\title{
Curative Effect Analysis of Zoledronic Acid in the Treatment of Postmenopausal Osteoporosis with Different Bone Turnover Rates
}

\author{
Suming Jie*, Jinmei Deng*, Caixia Lin, Xiuzhen Cai, Dongmei Cai\# \\ Outpatient Clinic of Internal Medicine Department, The First Affiliated Hospital of Sun Yat-sen University, \\ Guangzhou China \\ Email: jiesuming@126.com,309346496@qq.com, 15914399626@163.com, caixiuzhen66@126.com, "13500037898@163.com
}

How to cite this paper: Jie, S.M., Deng, J.M., Lin, C.X., Cai, X.Z. and Cai, D.M. (2018) Curative Effect Analysis of Zoledronic Acid in the Treatment of Postmenopausal Osteoporosis with Different Bone Turnover Rates. International Journal of Clinical Medicine, 9, 621-628.

https://doi.org/10.4236/ijcm.2018.98052

Received: July 6, 2018

Accepted: July 31, 2018

Published: August 3, 2018

Copyright $\odot 2018$ by authors and Scientific Research Publishing Inc. This work is licensed under the Creative Commons Attribution International License (CC BY 4.0).

http://creativecommons.org/licenses/by/4.0/

\begin{abstract}
Objective: To explore the clinical efficacy of Zoledronic Acid Injection in the treatment of postmenopausal osteoporosis with different bone turnover rates. Methods: A total of 63 patients diagnosed with postmenopausal osteoporosis were included in this study. Each patient was administrated $5 \mathrm{mg} / 100 \mathrm{~mL} \mathrm{Zo-}$ ledronic Acid (Aclasta) intravenously once and then given a one-year prescription of $600 \mathrm{mg} / \mathrm{d}$ oral Caltrate. The bone turnover parameters (PINP, $\beta$-cross, N-MID) were measured prior to the injection of Zoledronic Acid while the bone mineral density (BMD) and the pain scores of each patient were tested before treatment and after the one-year medication. On this basis, the patients were divided into several groups according to their bone turnover rates for intergroup comparison of treatment outcomes. Results: BMD results and pain scores of all participants were significantly improved at different levels after treatment. However, these improvements had no significant differences between the patients with high and low bone turnover rates. Conclusion: Zoledronic Acid Injection can relieve bone pain, enhance the quality of life and increase the BMD in patients with postmenopausal osteoporosis, regardless of the bone turnover status.
\end{abstract}

\section{Keywords}

Osteoporosis, Postmenopausal, Bone Turnover Rate, Zoledronic Acid

\section{Introduction}

Osteoporosis (OP) is commonly seen in the middle-aged and elderly population in China. It is caused by a variety of factors featuring a decrease in bone mass

*Authors contributed equally. 
and a deterioration in bone microarchitecture, which elevates the risk of fracture and fracture-prone metabolic bone diseases (MBDs). The clinical manifestations of OP mainly include pain, fracture, humpback and height loss. Considering the high mortality and disability rates, OP treatment and management has become a major issue in the public health sector [1]. Anti-osteoporosis drugs are gaining wider acceptance among clinicians treating OP. As an anti-osteoporosis drug, Zoledronic Acid (Aclasta) is Novartis' once-yearly injection that provides fracture protection in postmenopausal osteoporosis by inhibiting bone reabsorption and increasing bone mineral density (BMD) [2]. In addition, the bone turnover biomarkers released from bone remodeling, combined with the widely used BMD measurements, can improve the diagnostic accuracy of osteoporosis [3] [4]. The measurements above and bone turnover parameters are clinically applied to the analysis of the curative effect of Zoledronic Acid in the treatment of postmenopausal osteoporosis with different bone turnover rates.

\section{Materials and Methods}

\subsection{General Information}

The clinical data of 63 postmenopausal patients with osteoporosis who presented at our osteoporosis outpatient clinic from January 2016 to December 2017 were collected and analyzed in this study. All participants were postmenopausal women aged between 50 and 80 (mean age $=66.0 \pm 8.0$ ). Prior to the initial diagnosis, dual-energy X-ray absorptiometry (DXA) was used to test the bone mineral density of lumbar spine and femur, with the measured values being adopted as diagnosis and inclusion criteria. Patients were eligible for inclusion if the total lumbar spine (L1 - L4) bone mineral density value was no greater than -2.5 , or T-score was below -1.0 with preexisting fragility fractures, definite systemic symptoms such as back pain or height loss, and normal 25(OH)D. Exclusion criteria: 1) Previous use (within six months before treatment) of estrogen, glucocorticoid (GC), calcitonin and other bisphosphonates (BPs) that might influence bone metabolism; 2) diabetes and endocrine disorders of thyroid, parathyroid, adrenal gland or gonad; 3) secondary osteoporosis; 4) an allergy to Zoledronic Acid.

\subsection{Treatment Methods}

Zoledronic Acid (5 mg in $100 \mathrm{~mL}$ ready-to-infuse solution) is administrated intravenously, followed by the oral medication of $600 \mathrm{mg} / \mathrm{d}$ calcium carbonate D3 (Caltrate) for one year. The patients were required to get regular sun exposure, namely basking in the sun for half an hour between 11:00 and 15:00, twice a week. The Zoledronic Acid (Aclasta) prescribed for the patients is produced by Novartis. It is a once-yearly injection that provides fracture protection in postmenopausal osteoporosis.

\subsection{Outcome Measures}

The following parameters were documented: pre-treatment bone turnover pa- 
rameters (PINP, $\beta$-cross, N-MID), bone mineral density of lumbar spine and femur and bone pains before and after treatment. All bone turnover parameters were tested after an overnight fast by the clinical laboratory of our hospital; BMD was measured by dual-photon absorptiometry (DPA) and dual-energy $\mathrm{X}$-ray absorptiometry (DXA); during treatment, the patients were recommended to pay follow-up visits to the hospital to diagnose whether they had fresh fragility fractures.

In terms of bone pains, the Visual Analogue Scale (VAS) was used for pain assessment. VAS (4-point scale): 1) No pain; 2) Mild pain (without affecting sleep); 3) Moderate pain (affecting sleep at a tolerable degree); 4) Severe pain (intolerable, unable to fall asleep).

\subsection{Clinical Efficacy Assessment}

1) Bone pains and fresh fractures were tested after the one-year treatment.

2) The BMD $\left(\mathrm{g} / \mathrm{cm}^{2}\right)$ and VAS scores before and after treatment were documented and analyzed.

3) The patients were divided into different groups by each bone turnover parameter according to the corresponding normal range specified by our hospital (PINP: $0.00-36.4 \mathrm{ng} / \mathrm{ml} ; \beta$-cross: $0.30-2.00 \mathrm{ng} / \mathrm{ml}$; N-MID: $14.00-46.00$ $\mathrm{ng} / \mathrm{ml}$ ). Specifically, the patients were respectively divided into a normal PINP group and an abnormal group with higher PINP levels, a normal $\beta$-cross group and an abnormal one with lower $\beta$-cross levels, and a normal N-MID group and an abnormal one with lower N-MID levels to analyze the improvement in BMD and VAS score.

\subsection{Statistical Methods}

The statistical analysis software SPSS22.0 was used for data analysis. Measurement data were expressed in the form of "mean \pm standard deviation $(\mathrm{x} \pm \mathrm{s})$ " and the t-test was applied to the intragroup comparison. Besides, the chi-squared test was designed for the comparison of enumeration data. $\mathrm{P}<0.05$ indicated a difference of statistical significance.

\section{Results}

After being treated for one year, no fresh fractures were detected in the $63 \mathrm{pa}-$ tients during follow-up visits. As shown in Table 1, all intergroup $\mathrm{P}$ values are greater than 0.05 , that is, the intergroup differences show no statistical significance but comparability.

As shown in Table 2, the average post-treatment VAS score was significantly lower than the pre-treatment level, which indicates that the bone pains were alleviated in the course of treatment. Also, the BMD $\left(\mathrm{g} / \mathrm{cm}^{2}\right)$ of all patients was significantly improved after treatment. All this has proved the clinical efficacy of Zoledronic Acid to reduce bone pains and loss of bone mineral density. But Table 3 presents a statistical analysis based on the intergroup comparison of OP 
patients with different bone turnover rates, which indicates no statistically significant difference $(\mathrm{P}>0.05)$. In other words, Zoledronic Acid is efficacious in treating postmenopausal osteoporosis regardless of the bone turnover status.

Neither fresh fragility fractures nor obvious adverse reactions were detected in the participants throughout the course of treatment.

\section{Discussion}

Metabolic activity brings about dynamic changes and constant remodeling of bone tissue while $\mathrm{OP}$ is caused by an increase in osteoclasts and loss of BMD,

Table 1. Patients' general information by group.

\begin{tabular}{cccccccc}
\hline & $\begin{array}{c}\text { Number of } \\
\text { Cases }\end{array}$ & Age & $\begin{array}{c}\text { Pre-Treatment } \\
\mathrm{L}_{2-4} \text { BMD }\end{array}$ & $\begin{array}{c}\text { Pre-Treatment } \\
\text { VAS }\end{array}$ & P1 & P2 & P3 \\
\hline PINP $\rightarrow$ & 37 & $66.7 \pm 8.3$ & $0.827 \pm 0.153$ & $2.18 \pm 0.896$ & & & \\
PINP $\uparrow$ & 26 & $65.0 \pm 7.6$ & $0.858 \pm 0.110$ & $1.88 \pm 0.833$ & 0.401 & 0.376 & 0.18 \\
$\beta$-CROSS $\downarrow$ & 36 & $67.0 \pm 8.6$ & $0.842 \pm 0.150$ & $2.19 \pm 0.92$ & & & \\
$\beta$-CROSS $\rightarrow$ & 27 & $64.6 \pm 7.0$ & $0.836 \pm 0.119$ & $1.89 \pm 0.801$ & 0.248 & 0.868 & 0.173 \\
N-MID $\rightarrow$ & 32 & $67.5 \pm 8.5$ & $0844 \pm 0.142$ & $2.19 \pm 0.931$ & & & \\
N-MID $\uparrow$ & 31 & $64.4 \pm 7.3$ & $0.836 \pm 0.134$ & $1.94 \pm 0.814$ & 0.135 & 0.792 & 0.258 \\
\hline
\end{tabular}

Note: $\downarrow, \rightarrow$, and $\uparrow$ respectively denote subnormal, normal and above-normal levels of bone turnover parameters. P1, P2 and P3 respectively express the P-values based on the comparison of the age, pre-treatment $\mathrm{L}_{2-4} \mathrm{BMD}$, pre-treatment VAS between groups which divided by different levels of bone turnover parameters.

Table 2. Pre and post-treatment BMD and VAS scores.

\begin{tabular}{ccccc}
\hline & Number of Cases & $\begin{array}{c}\text { Before } \\
\text { Treatment }\end{array}$ & $\begin{array}{c}\text { After One-Year } \\
\text { Treatment }\end{array}$ & P-Value \\
\hline BMD $\left(\mathrm{g} / \mathrm{cm}^{2}\right)$ & 63 & $0.840 \pm 0.137$ & $0.883 \pm 0.151$ & 0.001 \\
VAS score & 63 & $2.06 \pm 0.878$ & $1.7 \pm 0.647$ & 0.026 \\
\hline
\end{tabular}

Table 3. Intergroup comparison of BMD increase, VAS (post-pre) based on different bone turnover rates.

\begin{tabular}{cccccc}
\hline & $\begin{array}{c}\text { Number of } \\
\text { Cases }\end{array}$ & $\begin{array}{c}\text { BMD Increase } \\
\left(\mathrm{g} / \mathrm{cm}^{2}\right)\end{array}$ & VAS (Post-Pre) & $\mathrm{P}_{1}$-Value & $\mathrm{P}_{2}$-Value \\
\hline $\mathrm{PINP} \rightarrow$ & 37 & $0.047 \pm 0.080$ & $-0.243 \pm 0.76$ & & \\
$\mathrm{PINP} \uparrow$ & 26 & $0.045 \pm 0.058$ & $-0.192 \pm 0.800$ & 0.096 & 0.799 \\
$\beta$-CROSS $\downarrow$ & 36 & $0.044 \pm 0.084$ & $-0.222 \pm 0.800$ & & 0.999 \\
$\beta$-CROSS $\rightarrow$ & 27 & $0.050 \pm 0.052$ & $-0.222 \pm 0.751$ & 0.744 & \\
$\mathrm{~N}-\mathrm{MID} \rightarrow$ & 32 & $0.051 \pm 0.087$ & $-0.219 \pm 0.792$ & & 0.971 \\
$\mathrm{~N}-\mathrm{MID} \uparrow$ & 31 & $0.036 \pm 0.050$ & $-0.226 \pm 0.762$ & 0.419 & \\
\hline
\end{tabular}

Note: $\mathrm{P}_{1}$-value, $\mathrm{P}_{2}$-value respectively express the $\mathrm{P}$-values based on the comparison of the BMD increase, VAS (post-pre) between groups which were divided by different levels of bone turnover parameters. 
which impedes bone matrix formation and calcium salts deposition and ultimately leads to increased bone resorption and imbalanced bone turnover-that is, bone resorption exceeds formation. In terms of the underlying pathogenesis, OP can be classified as primary and secondary. For the patients aged 60 and above, $56 \%$ have osteoporosis. Particularly, hip fractures contribute the greatest to morbidity and mortality among all osteoporotic fractures, with $20 \%$ of the patients died within a year after the hip fracture. With the population aging, osteoporosis becomes increasingly prevalent and imposes a national threat to public health. As such, anti-osteoporosis drugs are gaining wider clinical application. In addition, a great variety of anti-osteoporosis drugs targeting at different symptoms have been developed. For instance, Teriparatide works by stimulating new bone formation; BPs have an effect of inhibiting bone reabsorption [5].

It is well established clinically that BPs can prevent and treat OP by inhibiting osteoclast formation and bone reabsorption. The BP therapy is most often used to treat metabolic bone diseases (MBDs) featuring a significant increase in bone reabsorption, especially those with a high turnover rate [6]. Zoledronic Acid, as a new-generation bisphosphonate, spreads efficiently in bone tissue when given by injection into a vein. The high-affinity medication, first of all, is carried to the bone tissue with increased bone formation and reabsorption and selectively absorbed by active osteoclasts. It inhibits bone reabsorption by selective inhibition of osteoclast activity and induction of osteoclast apoptosis. Zoledronic Acid terminates the mevalonate pathway to block cell cycle, thereby inducing the apoptosis of osteoclasts and precursors of monocytes and inhibiting bone reabsorption. With an imidazole ring side chain with two nitrogen atoms, it shows a strong binding affinity to bone mineral and a longer action period [7] [8]. Zoledronic Acid Injection is presently known as the most effective medicine among all bone resorption inhibitors [9]. By comparing the bone reabsorption before and after treatment, Zoledronic Acid Injection is significantly effective in reducing the loss of BMD and bone pains. Further, Zoledronic Acid is administrated once a year and works for one year, which therefore notably increases patient compliance [10]. In a retrospective analysis of the efficacy of and the patient compliance with Zoledronic Acid for the treatment of osteoporosis, there were 5 men and 148 postmenopausal women with osteoporosis participating in the study and $66.5 \%$ of these participants had a history of fractures and showed poor compliance with treatment. All patients were given $5 \mathrm{mg} / 100 \mathrm{~mL}$ Zoledronic Acid intravenously and $85 \%$ received a second dose in the following year. The BMD was increased by $11 \%$ and $20.7 \%$ respectively after one and four years of treatment. Between the second and the third year of treatment, the $\beta$-cross value dropped significantly and fresh fractures were observed in only $10.4 \%$ of the participants, a marked decline in the occurrence of fractures. The therapy was so convenient that the patients showed higher compliance, which enhanced the management and clinical efficacy of OP treatment [11].

Metabolic products, namely bone metabolic markers, are released during bone 
remodeling. Bone metabolic markers are clinical indicators of bone turnover status. In clinical practices, these bone metabolic markers indicate the bone formation rate greater than the bone reabsorption rate, or vice versa. Type I procollagen-N-propeptide (PINP) is a bone formation marker reflecting the changes of newly synthesized type I collagen, namely an indicator of bone formation. The bone turnover marker N-terminal midfragment osteocalcin (N-MID OC) is a bone-specific, calcium binding protein released by bone formation and resorption. $\beta$-Cross, as a bone resorption marker, is the degradation product of type I collagen C-telopeptides. $\beta$-Cross, PINP and N-MID are three bone markers recommended by the International Osteoporosis Foundation (IOF). Zoledronic Acid is a representative bone resorption inhibitor. In a study investigating the effect of Zoledronic Acid Injection on osteoporosis, it was found that Zoledronic Acid Injection, combined with regular calcium and Vitamin D supplements, could effectively reduce the N-MID, b-CTX and PINP levels and improve the bone turnover rate; apart from this, the bone reabsorption parameter N-CTX decreased more drastically than the bone formation parameter PINP, indicating a significant improvement in quality of life [12]. For patients with different bone turnover rates, appropriate anti-osteoporosis drugs should be prescribed to inhibit bone reabsorption and accelerate bone formation [13]. Chao, M. et al. suggested that Zoledronic Acid could reduce bone turnover parameters in the treatment of osteoporosis, which means Zoledronic Acid is effective in inhibiting bone turnover [14].

As an increasing number of bone turnover parameters extend their clinical application, it becomes a cause for concern whether the clinical efficacy of Zoledronic Acid varies in the treatment of osteoporosis with different bone turnover rates. In this study, the patients were divided into several groups by different bone turnover parameters for intergroup comparison and the differences showed no statistical significance. The study results suggested no statistically significant differences in the curative effect of Zoledronic Acid on osteoporosis with different bone turnover rates. This indicates that bone turnover parameters are neither appropriate criteria for the clinical application of Zoledronic Acid, nor reliable indicators in the assessment of its curative effect. In terms of osteoporosis, an increase in the bone turnover rate means a high bone turnover and rapid bone loss, regardless of the relationship between bone formation and bone reabsorption. This, combined with the BMD test, serves as the basis for an initial or follow-up anti-osteoporosis therapy. If relevant bone turnover parameters rise abnormally, the patient probably has secondary osteoporosis.

\section{Limitations}

There were only 63 patients included in this study, and all patients were under observation for one year only. The results of the VAS are inevitably affected by the inherently subjective patients. Besides, the statistical analysis might be underrepresented because a relatively small number of patients were included in each group. 


\section{Conclusion}

Neither severe side effects nor fresh fractures occurred during the course of treatment. It was demonstrated that Zoledronic Acid is efficacious in the treatment of postmenopausal osteoporosis with different bone turnover rates by reducing the loss of BMD and bone pains in the patients without triggering serious side effects.

\section{Conflicts of Interest}

The authors declare no conflicts of interest regarding the publication of this paper.

\section{References}

[1] Huang, S., Lin, H., Zhu, X., et al. (2014) Zoledronic Acid Increases Bone Mineral Density and Improves Health-Related Quality of Life over Two Years of Treatment in Chinese Women with Postmenopausal Osteoporosis. Endokrynologia Polska, 65, 96-104. https://doi.org/10.5603/EP.2014.0014

[2] Gauthier, K., Bai, A., Perras, C., et al. (2012) Denosumab, Raloxifene, and Zoledronic Acid for the Treatment of Postmenopausal Osteoporosis: Clinical Effectiveness and Harms. Canadian Agency for Drugs and Technologies in Health, Ottawa, ON.

[3] Nishizawa, Y., Ohta, H., Miura, M., et al. (2013) Guidelines for the Use of Bone Metabolic Markers in the Diagnosis and Treatment of Osteoporosis. Journal of Bone and Mineral Metabolism, 31, 1-15. https://doi.org/10.1007/s00774-012-0392-y

[4] Cavalier, E., Bergmann, P., Bruyere, O., et al. (2016) The Role of Biochemical of Bone Turnover Markers in Osteoporosis and Metabolic Bone Disease: A Consensus Paper of the Belgian Bone Club. Osteoporosis International, 27, 2181-2195. https://doi.org/10.1007/s00198-016-3561-3

[5] Deal, C. (2009) Potential New Drug Targets for Osteoporosis. Nature Clinical Practice Rheumatology, 5, 20-27. https://doi.org/10.1038/ncprheum0977

[6] Ruza, I., Mirfakhraee, S., Orwoll, E., et al. (2013) Clinical Experience with Intravenous Zoledronic Acid in the Treatment of Male Osteoporosis: Evidence and Opinions. Therapeutic Advances in Musculoskeletal Disease, 5, 182-198. https://doi.org/10.1177/1759720X13485829

[7] Tasci, I., Safer, U., Cintosun, U., et al. (2016) Zoledronic Acid Use and Risk of Cognitive Decline among Elderly Women and Men with Osteoporosis. Endocrine, Metabolic \& Immune Disorders Drug Targets, 16, 32-38. https://doi.org/10.2174/1871530315666151104115829

[8] Gamsjaeger, S., Buchinger, B., Zwettler, E., et al. (2011) Bone Material Properties in Actively Bone-Forming Trabeculae in Postmenopausal Women with Osteoporosis after Three Years of Treatment with Once-Yearly Zoledronic Acid. Journal of Bone and Mineral Research, 26, 12-18. https://doi.org/10.1002/jbmr.180

[9] Sheedy, K.C., Camara, M.I. and Camacho, P.M. (2015) Comparison of the Efficacy, Adverse Effects, and Cost of Zoledronic Acid and Denosumab in the Treatment of Osteoporosis. Endocrine Practice, 21, 275-279. https://doi.org/10.4158/EP14106.OR

[10] Carmona, R. and Adachi, R. (2009) Treatment of Postmenopausal Osteoporosis, Patient Perspectives-Focus on Once Yearly Zoledronic Acid. Patient Preference and Adherence, 3, 189-193. https://doi.org/10.2147/PPA.S3494 
[11] Wang, C. (2017) Efficacy and Safety of Zoledronic Acid for Treatment of Postmenopausal Osteoporosis: A Meta-Analysis of Randomized Controlled Trials. American Journal of Therapeutics, 24, e544-e552.

[12] Cremers, S. and Garnero, P. (2006) Biochemical Markers of Bone Turnover in the Clinical Development of Drugs for Osteoporosis and Metastatic Bone Disease: Potential Uses and Pitfalls. Drugs, 66, 2031-2058.

https://doi.org/10.2165/00003495-200666160-00001

[13] Miura, M. (2013) Evaluation of Bone for Using of Bone Metabolic Markers in the Diagnosis and Treatment of Osteoporosis. Clinical Calcium, 23, 325-338.

[14] Chao, M., Hua, Q., Yingfeng, Z., et al. (2013) Study on the Role of Zoledronic Acid in Treatment of Postmenopausal Osteoporosis Women. Pakistan Journal of Medical Sciences, 29, 1381-1384. 